Applied Mathematical Sciences, Vol. 10, 2016, no. 9, 417 - 430

HIKARI Ltd, www.m-hikari.com

http://dx.doi.org/10.12988/ams.2016.510667

\title{
Generalized One-Step Third Derivative Implicit Hybrid Block Method for the Direct Solution of Second Order Ordinary Differential Equation
}

\author{
Z. Omar \\ Department of Mathematics \\ School of Quantitative Sciences \\ College of Art and Sciences \\ University Utara Malaysia, Malaysia \\ M. F. Alkasassbeh \\ Department of Mathematics \\ School of Quantitative Sciences \\ College of Art and Sciences \\ University Utara Malaysia, Malaysia
}

Copyright (c) 2015 Z. Omar and M. F. Alkasassbeh. This article is distributed under the Creative Commons Attribution License, which permits unrestricted use, distribution, and reproduction in any medium, provided the original work is properly cited.

\begin{abstract}
In this article, an implicit hybrid method of order six is developed for the direct solution of second order ordinary differential equations using collocation and interpolation approach. To derive this method, the approximate solution power series is interpolated at the first and off-step points and its second and third derivatives are collocated at all points in the given interval. Besides having good numerical method properties, the new developed method is also superior to the existing methods in terms of accuracy when solving the same problems.
\end{abstract}

Keywords: Collocation, Interpolation, Hybrid Block Methods, Second Order Ordinary Differential Equation, Direct Solution, Third derivative 


\section{Introduction}

This article proposes a general one-step third derivative implicit hybrid block method (GOHBM) for the direct solution of the second order ODEs in the form

$$
y^{\prime \prime}=f\left(x, y, y^{\prime}\right), \quad y(a)=y_{0}, \quad y_{0}^{\prime}(a)=y_{0}^{\prime}, \quad a \leqslant x \leqslant b
$$

with the assumption that $f$ is differentiable and satisfies Lipchitz's condition which guarantees the existence and uniqueness of the solution ([10]).

Block methods which are widely used by many scholars for solving (1) were first introduced by [14] and later by [9] mainly to provide starting values for predictor-corrector algorithms. Those methods produced better accuracy than the usual step by step methods. [8], on the other hand, extended Milne's idea to develop block methods for solving ODEs. In order to obtain higher order methods and hence to increase the accuracy of the approximate solution, [4] proposed hybrid block methods which included off-step point(s) in the derivation of the algorithms. Furthermore, hybrid block methods were used to circumvent Dahlquists barrier conditions which stipulate that the order of a k-step Linear Multistep Method (LMM) cannot exceed $k+1$ for $k$ is odd or $k+2$ for $k$ is even for the method to be zero-stable ([6]). In addition, hybrid block methods are also known to share with Runge-Kutta methods their favourable advantage of being self starting and more accurate since they are implemented as a block.

In hybrid block methods, step and off-step points are combined to form a single block for solving ODEs ( see [4], [15], [12]). In addition, [16] introduced second derivative methods which are special types of hybrid methods (referred by [14] as Obrechkoff methods) to enhance the accuracy of the approximation which shown to reach an order $k+2$. Meanwhile, some scholars such as [5], [11] proposed a Simpson's-type second derivative method for the solution of stiff system of first order IVPs. Their work motivated us to propose a new generalized one step third derivative implicit hybrid block method for solving second order ODEs directly using interpolation and collocation in the form

$\sum_{i=0}^{1} \alpha_{i t} y_{n+i t}=h^{2}\left[\sum_{i=0}^{1} \beta_{i t} f_{n+i t}+\beta_{1} f_{n+1}\right]+h^{3}\left[\sum_{i=0}^{1} \gamma_{i t} g_{n+i t}+\gamma_{1} g_{n+1}\right], \quad x \in\left[x_{n}, x_{n+1}\right]$

where $n=0,1,2, \ldots, N-1, h=x_{n}-x_{n-1}$ is the constant step size for the partition $\pi_{N}$ of the interval $[a, b]$ which is given by $\pi_{N}=\left[a=x_{0}<x_{1}<\ldots<\right.$ $\left.x_{N-1}<x_{N}=b\right], \alpha_{i t}, \beta_{i t}$ and $\gamma_{i t}$ are unknown coefficients, $g_{n+i t}=f_{n+i t}^{\prime}$ and $g_{n+1}=f_{n+1}^{\prime}$. 


\section{Development of the Method}

Let us assume the following power series be the approximate solution to (1)

$$
y(x)=\sum_{j=0}^{2 s+r-1} a_{j} x^{j}
$$

where $r$ and $s$ are the number of interpolation and collocation points respectively. Differentiating (2) twice and thrice yields

$$
\begin{gathered}
y^{\prime \prime}(x)=\sum_{j=2}^{2 s+r-1} a_{j} j(j-1) x^{j-2}=f\left(x, y, y^{\prime}\right) \\
y^{\prime \prime \prime}(x)=\sum_{j=3}^{2 s+r-1} a_{j} j(j-1)(j-2) x^{j-3}=g\left(x, y, y^{\prime}\right)
\end{gathered}
$$

Interpolating (2) at $x_{n+r}, r=\{0, t\}$ and collocating (3) and (4) at $x_{n+s}$, $s=\{0, t, 1\}$ where $t \in(0,1)$, and on combining gives a system of equations in matrix form

$$
A X=U
$$

where

$$
\begin{gathered}
A=\left[\begin{array}{llllllll}
a_{0} & a_{1} & a_{2} & a_{3} & a_{4} & a_{5} & a_{6} & a_{7}
\end{array}\right]^{T}, \\
U=\left[\begin{array}{llllllll}
y_{n} & y_{n+t} & f_{n} & f_{n+t} & f_{n+1} & g_{n} & g_{n+t} & g_{n+1}
\end{array}\right]^{T},
\end{gathered}
$$

and

$$
X=\left[\begin{array}{cccccccc}
1 & x_{n} & x_{n}^{2} & x_{n}^{3} & x_{n}^{4} & x_{n}^{5} & x_{n}^{6} & x_{n}^{7} \\
1 & x_{n+t} & x_{n+t}^{2} & x_{n+t}^{3} & x_{n+t}^{4} & x_{n+t}^{5} & x_{n+t}^{6} & x_{n+t}^{7} \\
0 & 0 & 2 & 6 x_{n} & 12 x_{n}^{2} & 20 x_{n}^{3} & 30 x_{n}^{4} & 42 x_{n}^{5} \\
0 & 0 & 2 & 6 x_{n+t} & 12 x_{n+t}^{2} & 20 x_{n+t}^{3} & 30 x_{n+t}^{4} & 42 x_{n+t}^{5} \\
0 & 0 & 2 & 6 x_{n+1} & 12 x_{n+1}^{2} & 20 x_{n+1}^{3} & 30 x_{n+1}^{4} & 42 x_{n+1}^{5} \\
0 & 0 & 0 & 6 & 24 x_{n} & 60 x_{n}^{2} & 120 x_{n}^{3} & 210 x_{n}^{4} \\
0 & 0 & 0 & 6 & 24 x_{n+t} & 60 x_{n+t}^{3} & 120 x_{n+t}^{3} & 210 x_{n+t}^{4} \\
0 & 0 & 0 & 6 & 24 x_{n+1} & 60 x_{n+1}^{2} & 120 x_{n+1}^{3} & 210 x_{n+1}^{4}
\end{array}\right]
$$

Solving (5) for the unknown constant $a_{j}^{\prime} s$ using matrix manipulation and substituting them back into (2) gives a continuous hybrid linear multi-step method in the form

$$
y(x)=\sum_{i=0}^{1} \alpha_{i t} y_{n+i t}+h^{2}\left[\sum_{i=0}^{1} \beta_{i t} f_{n+i t}+\beta_{1} f_{n+1}\right]+h^{3}\left[\sum_{i=0}^{1} \gamma_{i t} g_{n+i t}+\gamma_{1} g_{n+1}\right]
$$


whose first derivative is

$$
\left.y^{\prime}(x)=\frac{1}{h} \sum_{i=0}^{1} \alpha_{i t}^{\prime} y_{n+i t}+h\left[\sum_{i=0}^{1} \beta_{i t}^{\prime} f_{n+i t}+\beta_{1}^{\prime} f_{n+1}\right]+h^{2} \sum_{i=0}^{1} \gamma_{i t}^{\prime} g_{n+i t}+\gamma_{1}^{\prime} g_{n+1}\right]
$$

Evaluating (6) at $x=x_{n+1}$ and (7) at $x=x_{n+i}, i=\{0, t, 1\}$ produces the following general equations in block form

$$
A^{(0)} Y_{m+1}=A^{(t)} Y_{m}+\sum_{i=0}^{1} B^{(i)} F_{m+i}+\sum_{i=0}^{1} D^{(i)} G_{m+i}
$$

where $A^{(0)}$ is a $4 \times 4$ identity matrix, $Y_{m+1}=\left[y_{n+t}, y_{n+1}, y_{n+t}^{\prime}, y_{n+1}^{\prime}\right]^{T}, Y_{m}=$ $\left[y_{n-t}, y_{n}, y_{n-t}^{\prime}, y_{n}^{\prime}\right]^{T}$,

$F_{m}=\left[f_{n-3 t}, f_{n-2 t}, f_{n-t}, f_{n}\right]^{T}, F_{m+1}=\left[f_{n+t}, f_{n+1}\right]^{T}, G_{m}=\left[g_{n-3 t}, g_{n-2 t}, g_{n-t}, g_{n}\right]^{T}$, $G_{m+1}=\left[g_{n+t}, g_{n+1}\right]^{T}$.

The matrices $A^{(t)}, B^{(i)}, D^{(i)}$ will be described later. To obtain the specific equations of (8), let us consider the following three cases for demonstration.

Case I : $t=\frac{1}{3}$

Substituting $t=\frac{1}{3}$ and $z=\frac{x-x_{n+\frac{1}{3}}}{h}$ in (6) and (7) we get

$$
y(z)=\sum_{i=0}^{1} \alpha_{\frac{i}{3}} y_{n+\frac{i}{3}}+h^{2}\left[\sum_{i=0}^{1} \beta_{\frac{i}{3}} f_{n+\frac{i}{3}}+\beta_{1} f_{n+1}\right]+h^{3}\left[\sum_{i=0}^{1} \gamma_{\frac{i}{3}} g_{n+\frac{i}{3}}+\gamma_{1} g_{n+1}\right]
$$

where

$$
\begin{aligned}
\alpha_{0} & =-3 z \\
\alpha_{\frac{1}{3}} & =1+3 z \\
\beta_{0} & =\left(z(1+3 z)\left(709-2127 z+6381 z^{2}+1647 z^{3}-15147 z^{4}+9720 z^{5}\right)\right) / 17010 \\
\beta_{\frac{1}{3}} & =-\left(\left(z(1+3 z)\left(-626-642 z+1926 z^{2}+837 z^{3}-4212 z^{4}+2430 z^{5}\right)\right) / 5040\right) \\
\beta_{1} & =-\left(\left(z(1+3 z)\left(-106+318 z-954 z^{2}-9423 z^{3}-7452 z^{4}+12150 z^{5}\right)\right) / 136080\right) \\
\gamma_{0} & =\left(z(1+3 z)\left(44-132 z+396 z^{2}+702 z^{3}-2106 z^{4}+1215 z^{5}\right)\right) / 17010 \\
\gamma_{\frac{1}{3}} & =\left(z(1+3 z)\left(-115+345 z+1485 z^{2}-675 z^{3}-3078 z^{4}+2430 z^{5}\right)\right) / 15120 \\
\gamma_{1} & =\left(z(1+3 z)\left(-17+51 z-153 z^{2}-1431 z^{3}-810 z^{4}+2430 z^{5}\right)\right) / 136080
\end{aligned}
$$


and

$$
\left.y^{\prime}(z)=\frac{1}{h} \sum_{i=0}^{1} \alpha_{\frac{i}{3}}^{\prime} y_{n+\frac{i}{3}}+h\left[\sum_{i=0}^{1} \beta_{\frac{i}{3}}^{\prime} f_{n+\frac{i}{3}}+\beta_{1}^{\prime} f_{n+1}\right]+h^{2} \sum_{i=0}^{1} \gamma_{i t}^{\prime} g_{n+\frac{i}{3}}+\gamma_{1}^{\prime} g_{n+1}\right]
$$

where

$$
\begin{aligned}
\alpha_{0}^{\prime} & =-3 \\
\alpha_{1}^{\prime} & =3 \\
\beta_{0}^{\prime} & =\left(709+83160 z^{3}-51030 z^{4}-214326 z^{5}+204120 z^{6}\right) / 17010 \\
\beta_{\frac{1}{3}}^{\prime} & =\left(626+5040 z-26460 z^{3}+8505 z^{4}+61236 z^{5}-51030 z^{6}\right) / 5040 \\
\beta_{1}^{\prime} & =\left(106+49140 z^{3}+178605 z^{4}+61236 z^{5}-255150 z^{6}\right) / 136080 \\
\gamma_{0}^{\prime} & =\left(44+7560 z^{3}-30618 z^{5}+25515 z^{6}\right) / 17010 \\
\gamma_{\frac{1}{3}}^{\prime} & =\left(-115+7560 z^{2}+15120 z^{3}-25515 z^{4}-40824 z^{5}+51030 z^{6}\right) / 15120 \\
\gamma_{1}^{\prime} & =\left(-17-7560 z^{3}-25515 z^{4}+51030 z^{6}\right) / 136080
\end{aligned}
$$

Now, equation (8) can be written as

$$
A^{(0)} Y_{m+1}=A^{\left(\frac{1}{3}\right)} Y_{m}+\sum_{i=0}^{1} B^{(i)} F_{m+i}+\sum_{i=0}^{1} D^{(i)} G_{m+i}
$$

where

$$
\begin{gathered}
A^{\left(\frac{1}{3}\right)}=\left[\begin{array}{llll}
0 & 1 & 0 & \frac{h}{3} \\
0 & 1 & 0 & h \\
0 & 0 & 0 & 1 \\
0 & 0 & 0 & 1
\end{array}\right] B^{(0)}=\left[\begin{array}{cccc}
0 & 0 & 0 & \frac{613 h^{2}}{17010} \\
0 & 0 & 0 & \frac{3 h^{2}}{14} \\
0 & 0 & 0 & \frac{182 h}{1215} \\
0 & 0 & 0 & \frac{2 h}{5}
\end{array}\right] B^{(1)}=\left[\begin{array}{cc}
\frac{97 h^{2}}{5040} & \frac{37 h^{2}}{136080} \\
\frac{27 h^{2}}{112} & \frac{5 h^{2}}{112} \\
\frac{131 h}{720} & \frac{31 h}{1940} \\
\frac{27 h}{80} & \frac{21 h}{80}
\end{array}\right] \\
D^{(0)}=\left[\begin{array}{llll}
0 & 0 & 0 & \frac{5 h^{3}}{3402} \\
0 & 0 & 0 & \frac{h^{3}}{70} \\
0 & 0 & 0 & \frac{17 h^{2}}{2430} \\
0 & 0 & 0 & \frac{h^{2}}{30}
\end{array}\right] D^{(1)}=\left[\begin{array}{cc}
\frac{-11 h^{3}}{5670} & \frac{-h^{3}}{22680} \\
\frac{9 h^{3}}{280} & \frac{-h^{3}}{210} \\
\frac{-29 h^{2}}{2160} & \frac{-h^{2}}{3888} \\
\frac{9 h^{2}}{80} & \frac{-h^{2}}{48}
\end{array}\right]
\end{gathered}
$$

Case II : $t=\frac{1}{2}$

Similarly, replacing $t=\frac{1}{2}$ and $z=\frac{x-x_{n+\frac{1}{2}}}{h}$ in (6) and (7) produces

$$
y(z)=\sum_{i=0}^{1} \alpha_{\frac{i}{2}} y_{n+\frac{i}{2}}+h^{2}\left[\sum_{i=0}^{1} \beta_{\frac{i}{2}} f_{n+\frac{i}{2}}+\beta_{1} f_{n+1}\right]+h^{3}\left[\sum_{i=0}^{1} \gamma_{\frac{i}{2}} g_{n+\frac{i}{2}}+\gamma_{1} g_{n+1}\right]
$$


where

$$
\begin{aligned}
\alpha_{0} & =-2 z \\
\alpha_{\frac{1}{2}} & =1+2 z \\
\beta_{0} & =\left(z(2 z+1)\left(960 z^{5}-928 z^{4}-376 z^{3}+748 z^{2}-374 z+187\right)\right) / 3360 \\
\beta_{\frac{1}{2}} & =z\left(32 z^{5}-40 z^{3}+30 z+11\right) / 60 \\
\beta_{1} & =-\left(\left(z(2 z+1)\left(960 z^{5}-32 z^{4}-824 z^{3}-148 z^{2}+74 z-37\right)\right) / 3360\right) \\
\gamma_{0} & =z(2 z+1)\left(40 z^{5}-48 z^{4}+3 z^{3}+16 z^{2}-8 z+4\right) / 840 \\
\gamma_{\frac{1}{2}} & =z\left(320 z^{6}-336 z^{4}+140 z^{2}-19\right) / 840 \\
\gamma_{1} & =\left(z\left(320 z^{6}+224 z^{5}-168 z^{4}-140 z^{3}-5\right)\right) / 3360
\end{aligned}
$$

and

$$
\left.y^{\prime}(z)=\frac{1}{h} \sum_{i=0}^{1} \alpha_{\frac{i}{2}}^{\prime} y_{n+\frac{i}{2}}+h\left[\sum_{i=0}^{1} \beta_{\frac{i}{2}}^{\prime} f_{n+\frac{i}{3}}+\beta_{1}^{\prime} f_{n+1}\right]+h^{2} \sum_{i=0}^{1} \gamma_{\frac{i}{2}}^{\prime} g_{n+\frac{i}{2}}+\gamma_{1}^{\prime} g_{n+1}\right]
$$

where

$$
\begin{aligned}
\alpha_{0}^{\prime} & =-2 \\
\alpha_{1}^{\prime} & =2 \\
\beta_{0}^{\prime} & =\left(13440 z^{6}-5376 z^{5}-8400 z^{4}+4480 z^{3}+187\right) / 3360 \\
\beta_{\frac{1}{2}}^{\prime} & =\left(192 z^{5}-160 z^{3}+60 z+11\right) / 60 \\
\beta_{1}^{\prime} & =\left(-13440 z^{6}-5376 z^{5}+8400 z^{4}+4480 z^{3}+37\right) / 3360 \\
\gamma_{0}^{\prime} & =\left(280 z^{6}-168 z^{5}-105 z^{4}+70 z^{3}+2\right) / 420 \\
\gamma_{\frac{1}{2}}^{\prime} & =\left(2240 z^{6}-1680 z^{4}+420 z^{2}-19\right) / 840 \\
\gamma_{1}^{\prime} & =\left(2240 z^{6}+1344 z^{5}-840 z^{4}-560 z^{3}-5\right) / 3360
\end{aligned}
$$

Thus, equation (8) becomes

$$
A^{(0)} Y_{m+1}=A^{\left(\frac{1}{2}\right)} Y_{m}+\sum_{i=0}^{1} B^{(i)} F_{m+i}+\sum_{i=0}^{1} D^{(i)} G_{m+i}
$$

where

$$
A^{\left(\frac{1}{2}\right)}=\left[\begin{array}{llll}
0 & 1 & 0 & \frac{h}{2} \\
0 & 1 & 0 & h \\
0 & 0 & 0 & 1 \\
0 & 0 & 0 & 1
\end{array}\right] B^{(0)}=\left[\begin{array}{cccc}
0 & 0 & 0 & \frac{13 h^{2}}{168} \\
0 & 0 & 0 & \frac{79 h^{2}}{420} \\
0 & 0 & 0 & \frac{101 h}{480} \\
0 & 0 & 0 & \frac{7 h}{30}
\end{array}\right] B^{(1)}=\left[\begin{array}{cc}
\frac{h^{2}}{24} & \frac{h^{2}}{168} \\
\frac{4 h^{2}}{15} & \frac{79 h^{2}}{420} \\
\frac{4 h}{15} & \frac{101 h}{480} \\
\frac{8 h}{15} & \frac{7 h}{30}
\end{array}\right]
$$




$$
D^{(0)}=\left[\begin{array}{cccc}
0 & 0 & 0 & \frac{59 h^{3}}{13440} \\
0 & 0 & 0 & \frac{h^{3}}{84} \\
0 & 0 & 0 & \frac{13 h^{2}}{960} \\
0 & 0 & 0 & \frac{h^{2}}{60}
\end{array}\right] \quad D^{(1)}=\left[\begin{array}{cc}
\frac{-h^{3}}{105} & \frac{-11 h^{3}}{1340} \\
\frac{-2 h^{3}}{105} & \frac{-h^{3}}{210} \\
\frac{-h^{2}}{24} & \frac{-h^{2}}{320} \\
0 & \frac{-h^{2}}{60}
\end{array}\right]
$$

Case III : $t=\frac{2}{3}$

Finally, putting $t=\frac{2}{3}$ and $z=\frac{x-x_{n+\frac{2}{3}}}{h}$ in (6) and (7) we have

$$
y(z)=\sum_{i=0}^{1} \alpha_{\frac{2 i}{3}} y_{n+\frac{2 i}{3}}+h^{2}\left[\sum_{i=0}^{1} \beta_{\frac{2 i}{3}} f_{n+\frac{2 i}{3}}+\beta_{1} f_{n+1}\right]+h^{3}\left[\sum_{i=0}^{1} \gamma_{\frac{2 i}{3}} g_{n+\frac{2 i}{3}}+\gamma_{1} g_{n+1}\right]
$$

where

$$
\begin{aligned}
\alpha_{0} & =-3 z / 2 \\
\alpha_{\frac{2}{3}} & =1+3 z / 2 \\
\beta_{0} & =\left(z(2+3 z)\left(4420-6630 z+9945 z^{2}-8775 z^{3}-4698 z^{4}+12150 z^{5}\right)\right) / 136080 \\
\beta_{\frac{2}{3}} & =\left(z(2+3 z)\left(380+690 z-1035 z^{2}-1755 z^{3}+1782 z^{4}+2430 z^{5}\right)\right) / 5040 \\
\beta_{1} & =-\left(\left(z(2+3 z)\left(-1000+1500 z-2250 z^{2}-7020 z^{3}+5427 z^{4}+9720 z^{5}\right)\right) / 17010\right) \\
\gamma_{0} & =\left(z(2+3 z)\left(464-696 z+1044 z^{2}-621 z^{3}-1620 z^{4}+2430 z^{5}\right)\right) / 136080 \\
\gamma_{\frac{2}{3}} & =\left(z(2+3 z)\left(-488+732 z+162 z^{2}-2133 z^{3}+648 z^{4}+2430 z^{5}\right)\right) / 15120 \\
\gamma_{1} & =\left(z(2+3 z)\left(-104+156 z-234 z^{2}-594 z^{3}+891 z^{4}+1215 z^{5}\right)\right) / 17010
\end{aligned}
$$

and

$$
\left.y^{\prime}(z)=\frac{1}{h} \sum_{i=0}^{1} \alpha_{\frac{2 i}{3}}^{\prime} y_{n+\frac{2 i}{3}}+h\left[\sum_{i=0}^{1} \beta_{\frac{2 i}{3}}^{\prime} f_{n+\frac{2 i}{3}}+\beta_{1}^{\prime} f_{n+1}\right]+h^{2} \sum_{i=0}^{1} \gamma_{\frac{2 i}{3}}^{\prime} g_{n+\frac{2 i}{3}}+\gamma_{1}^{\prime} g_{n+1}\right]
$$

where

$$
\begin{aligned}
\alpha_{0}^{\prime} & =-3 / 2 \\
\alpha_{1}^{\prime} & =3 / 2 \\
\beta_{0}^{\prime} & =\left(8840+49140 z^{3}-178605 z^{4}+61236 z^{5}+255150 z^{6}\right) / 136080 \\
\beta_{\frac{2}{3}}^{\prime} & =\left(760+5040 z-26460 z^{3}-8505 z^{4}+61236 z^{5}+51030 z^{6}\right) / 5040 \\
\beta_{1}^{\prime} & =\left(1000+41580 z^{3}+25515 z^{4}-107163 z^{5}-102060 z^{6}\right) / 8505 \\
\gamma_{0}^{\prime} & =\left(928+7560 z^{3}-25515 z^{4}+51030 z^{6}\right) / 136080 \\
\gamma_{\frac{2}{3}}^{\prime} & =\left(-976+7560 z^{2}-15120 z^{3}-25515 z^{4}+40824 z^{5}+51030 z^{6}\right) / 15120 \\
\gamma_{1}^{\prime} & =\left(-208-7560 z^{3}+30618 z^{5}+25515 z^{6}\right) / 17010
\end{aligned}
$$


Hence, we can write equation (8) as below

$$
A^{(0)} Y_{m+1}=A^{\left(\frac{2}{3}\right)} Y_{m}+\sum_{i=0}^{1} B^{(i)} F_{m+i}+\sum_{i=0}^{1} D^{(i)} G_{m+i}
$$

where

$$
\begin{gathered}
A^{\left(\frac{2}{3}\right)=}\left[\begin{array}{llll}
0 & 1 & 0 & \frac{2 h}{3} \\
0 & 1 & 0 & h \\
0 & 0 & 0 & 1 \\
0 & 0 & 0 & 1
\end{array}\right] B^{(0)}=\left[\begin{array}{llll}
0 & 0 & 0 & \frac{1111 h^{2}}{8505} \\
0 & 0 & 0 & \frac{61 h^{2}}{280} \\
0 & 0 & 0 & \frac{317 h}{1215} \\
0 & 0 & 0 & \frac{21 h}{80}
\end{array}\right] B^{(1)}=\left[\begin{array}{cc}
\frac{h^{2}}{315} & \frac{752 h^{2}}{8505} \\
\frac{27 h^{2}}{280} & \frac{13 h^{2}}{70} \\
\frac{7 h}{45} & \frac{304 h}{1215} \\
\frac{27 h}{80} & \frac{2 h}{5}
\end{array}\right] \\
D^{(0)}=\left[\begin{array}{llll}
0 & 0 & 0 & \frac{26 h^{3}}{2835} \\
0 & 0 & 0 & \frac{9 h^{3}}{560} \\
0 & 0 & 0 & \frac{5 h^{2}}{243} \\
0 & 0 & 0 & \frac{h^{2}}{48}
\end{array}\right] D^{(1)}=\left[\begin{array}{cc}
\frac{-116 h^{3}}{2835} & \frac{-16 h^{3}}{1701} \\
\frac{-9 h^{3}}{112} & \frac{-2 h^{3}}{105} \\
\frac{-17 h^{2}}{135} & \frac{-32 h^{2}}{1215} \\
\frac{-9 h^{3}}{80} & \frac{-h^{2}}{30}
\end{array}\right]
\end{gathered}
$$

\section{Analysis of the Method}

\section{Order of the method}

The linear operator $\hat{L}$ associated with the hybrid block methods formula (8) according to [13] and [7] is said to be of order $p$ if

$$
\hat{L}\{y(x) ; h\}=A^{(0)} Y_{m}-A^{(t)} Y_{m+1}-\sum_{i=0}^{1} B^{(i)} F_{m+i}-\sum_{i=0}^{1} D^{(i)} G_{m+i}
$$

expanding in Taylor series and combining like terms

$$
\hat{L}\{y(x) ; h\}=\sum_{i=0}^{\infty} C_{i} h^{i} y^{(i)}=0
$$

where

$$
C_{0}=C_{1}=\ldots=C_{p+1}=0 \text { and } C_{p+2} \neq 0
$$

The term $C_{p+2}$ is called the error constant and the local truncation error is given by :

$$
t_{n+k}=C_{p+2} y^{p+2} h^{p+2}\left(x_{n}\right)+O\left(h^{p+3}\right)
$$

For Case (I), substituting $t=\frac{1}{3}$ in (9), we get 


$$
\left[\begin{array}{c}
\sum_{i=0}^{\infty}\left(\frac{h^{i}}{3^{i} i !}\right) y_{n}^{(i)}-y_{n}-\frac{h}{3} y_{n}^{\prime}-\frac{613 h^{2} y_{n}^{(2)}}{17010}-\sum_{i=0}^{\infty} \frac{h^{i+2} y_{n}^{(i+2)}}{136080 i !}\left(2619\left(\frac{1}{3}\right)^{i}+37\right)-\frac{5 h^{3} y_{n}^{(3)}}{3402} \\
+\sum_{i=0}^{\infty} \frac{h^{i+3} y_{n}^{(i+3)}}{22680 i !}\left(44\left(\frac{1}{3}\right)^{i}+1\right) \\
\sum_{i=0}^{\infty}\left(\frac{h^{i}}{i !}\right) y_{n}^{(i)}-y_{n}-h y_{n}^{\prime}-\frac{3 h^{2} y_{n}^{(2)}}{14}-\sum_{i=0}^{\infty} \frac{h^{i+2} y_{n}^{(i+2)}}{112 i !}\left(27\left(\frac{1}{3}\right)^{i}+5\right)-\frac{h^{3} y_{n}^{(3)}}{70} \\
-\sum_{i=0}^{\infty} \frac{h^{i+3} y_{n}^{(i+3)}}{840 i !}\left(27\left(\frac{1}{3}\right)^{i}-4\right) \\
\sum_{i=0}^{\infty}\left(\frac{h^{i}}{3^{i} i !}\right) y_{n}^{(i+1)}-y_{n}^{\prime}-\frac{182 h y_{n}^{(2)}}{1215}-\sum_{i=0}^{\infty} \frac{h^{i+1} y_{n}^{(i+2)}}{19440 i !}\left(3537\left(\frac{1}{3}\right)^{i}+31\right)-\frac{17 h^{2} y_{n}^{(3)}}{2430} \\
+\sum_{i=0}^{\infty} \frac{h^{i+2} y_{n}^{(i+3)}}{19440 i !}\left(261\left(\frac{1}{3}\right)^{i}+5\right) \\
\sum_{i=0}^{\infty}\left(\frac{h^{i}}{i !}\right) y_{n}^{(i+1)}-y_{n}^{\prime}-\frac{2 h y_{n}^{(2)}}{5}-\sum_{i=0}^{\infty} \frac{h^{i+1} y_{n}^{(i+2)}}{80 i !}\left(27\left(\frac{1}{3}\right)^{i}+21\right)-\frac{h^{i+2} y_{n}^{(3)}}{30} \\
-\sum_{i=0}^{\infty} \frac{h^{i+2} y_{n}^{(i+3)}}{240 i !}\left(27\left(\frac{1}{3}\right)^{i}-5\right)
\end{array}\right]=\left[\begin{array}{c}
0 \\
0
\end{array}\right]=\left[\begin{array}{l}
0 \\
0
\end{array}\right]
$$

Comparing the coefficients of $y^{i}$ and $h^{i}$ produces $C_{0}=C_{1}=\ldots=C_{7}=0$ with vector of error constants

$C_{8}=\left[\begin{array}{llll}\frac{31}{1322697600} & \frac{1}{1088640} & \frac{11}{82668600} & \frac{1}{340200}\end{array}\right]^{T}$ which implies the order $(p)$ of this method is 6 .

For Case (II), substituting $t=\frac{1}{2}$ in (9), we have

$$
\left[\begin{array}{c}
\sum_{i=0}^{\infty}\left(\frac{h^{i}}{2^{i} i !}\right) y_{n}^{(i)}-y_{n}-\frac{h}{2} y_{n}^{\prime}-\frac{13 h^{2} y_{n}^{(2)}}{168}-\sum_{i=0}^{\infty} \frac{h^{i+2} y_{n}^{(i+2)}}{168 i !}\left(7\left(\frac{1}{2}\right)^{i}+1\right)-\frac{59 h^{3} y_{n}^{(3)}}{13440} \\
+\sum_{i=0}^{\infty} \frac{h^{i+3} y_{n}^{(i+3)}}{13440 i !}\left(128\left(\frac{1}{2}\right)^{i}+11\right) \\
\sum_{i=0}^{\infty}\left(\frac{h^{i}}{i !}\right) y_{n}^{(i)}-y_{n}-h y_{n}^{\prime}-\frac{79 h^{2} y_{n}^{(2)}}{420}-\sum_{i=0}^{\infty} \frac{h^{i+2} y_{n}^{(i+2)}}{420 i !}\left(112\left(\frac{1}{2}\right)^{i}+19\right)-\frac{5 h^{3} y_{n}^{(3)}}{420} \\
+\sum_{i=0}^{\infty} \frac{h^{i+3} y_{n}^{(i+3)}}{420 i !}\left(8\left(\frac{1}{2}\right)^{i}+2\right) \\
\sum_{i=0}^{\infty}\left(\frac{h^{i}}{2^{i} i !}\right) y_{n}^{(i+1)}-y_{n}^{\prime}-\frac{101 h y_{n}^{(2)}}{420}-\sum_{i=0}^{\infty} \frac{h^{i+1} y_{n}^{(i+2)}}{420 i !}\left(128\left(\frac{1}{2}\right)^{i}+11\right)-\frac{13 h^{2} y_{n}^{(3)}}{960} \\
+\sum_{i=0}^{\infty} \frac{h^{i+2} y_{n}^{(i+3)}}{960 i !}\left(40\left(\frac{1}{2}\right)^{i}+3\right) \\
\sum_{i=0}^{\infty}\left(\frac{h^{i}}{i !}\right) y_{n}^{(i+1)}-y_{n}^{\prime}-\frac{7 h y_{n}^{(2)}}{30}-\sum_{i=0}^{\infty} \frac{h^{i+1} y_{n}^{(i+2)}}{30 i !}\left(16\left(\frac{1}{2}\right)^{i}+7\right)-\frac{h^{i+2} y_{n}^{(3)}}{60} \\
+\sum_{i=0}^{\infty} \frac{h^{i+2} y_{n}^{(i+3)}}{60 i !}
\end{array}\right]=\left[\begin{array}{l}
0 \\
0 \\
0
\end{array}\right]
$$

Associating the coefficients of $y^{i}$ and $h^{i}$ yields $C_{0}=C_{1}=\ldots=C_{7}=0$ with vector of error constants

$C_{8}=\left[\begin{array}{llll}\frac{1}{4423680} & \frac{1}{1209600} & \frac{1}{1209600} & \frac{1}{604800}\end{array}\right]^{T}$ which also implies that the order $(p)$ of this method is 6 . 
For Case (III), substituting $t=\frac{2}{3}$ in (9), we get

$$
\left[\begin{array}{c}
\sum_{i=0}^{\infty}\left(\frac{(2 h)^{i}}{3^{i} i !}\right) y_{n}^{(i)}-y_{n}-\frac{2 h}{3} y_{n}^{\prime}-\frac{1111 h^{2} y_{n}^{(2)}}{8505}-\sum_{i=0}^{\infty} \frac{h^{i+2} y_{n}^{(i+2)}}{8505 i !}\left(27\left(\frac{2}{3}\right)^{i}+752\right)-\frac{26 h^{3} y_{n}^{(3)}}{2835} \\
+\sum_{i=0}^{\infty} \frac{4 h^{i+3} y_{n}^{(i+3)}}{8505 i !}\left(87\left(\frac{2}{3}\right)^{i}+20\right) \\
\sum_{i=0}^{\infty}\left(\frac{h^{i}}{i !}\right) y_{n}^{(i)}-y_{n}-h y_{n}^{\prime}-\frac{61 h^{2} y_{n}^{(2)}}{280}-\sum_{i=0}^{\infty} \frac{h^{i+2} y_{n}^{(i+2)}}{280 i !}\left(27\left(\frac{2}{3}\right)^{i}+52\right)-\frac{9 h^{3} y_{n}^{(3)}}{560} \\
+\sum_{i=0}^{\infty} \frac{h^{i+3} y_{n}^{(i+3)}}{1680 i !}\left(135\left(\frac{2}{3}\right)^{i}+32\right) \\
\sum_{i=0}^{\infty}\left(\frac{(2 h)^{i}}{3^{i} i !}\right) y_{n}^{(i+1)}-y_{n}^{\prime}-\frac{317 h y_{n}^{(2)}}{1215}-\sum_{i=0}^{\infty} \frac{h^{i+1} y_{n}^{(i+2)}}{1215 i !}\left(189\left(\frac{2}{3}\right)^{i}+304\right)-\frac{5 h^{2} y_{n}^{(3)}}{243} \\
+\sum_{i=0}^{\infty} \frac{h^{i+2} y_{n}^{(i+3)}}{1215 i !}\left(153\left(\frac{2}{3}\right)^{i}+32\right) \\
\sum_{i=0}^{\infty}\left(\frac{h^{i}}{i !}\right) y_{n}^{(i+1)}-y_{n}^{\prime}-\frac{21 h y_{n}^{(2)}}{80}-\sum_{i=0}^{\infty} \frac{h^{i+1} y_{n}^{(i+2)}}{80 i !}\left(27\left(\frac{2}{3}\right)^{i}+32\right)-\frac{h^{i+2} y_{n}^{(3)}}{48} \\
+\sum_{i=0}^{\infty} \frac{h^{i+2} y_{n}^{(i+3)}}{240 i !}\left(27\left(\frac{2}{3}\right)^{i}+8\right)
\end{array}\right]=\left[\begin{array}{c}
0 \\
0
\end{array}\right]
$$

Matching the coefficients of $y^{i}$ and $h^{i}$ yields $C_{0}=C_{1}=\ldots=C_{7}=0$ with vector of error constants

$C_{8}=\left[\begin{array}{llll}\frac{11}{10333575} & \frac{11}{5443200} & \frac{29}{10333575} & \frac{1}{340200}\end{array}\right]^{T}$ which again implies that the order $(p)$ of this method is 6 .

\subsection{Consistency}

Definition 3.1. A block method is said to be consistent if its order is greater than one.

We conclude from the three cases above that the order $(p)$ of the hybrid block methods formula (8) is greater than 1 hence the consistency property is satisfied.

\subsection{Zero Stability}

Definition 3.2. The hybrid block method formula (8) is said to be zero stable if no root of the first characteristic equation $\rho(R)$ has modulus greater than one i.e $\left|R_{s}\right| \leqslant 1$ and if $R_{s}=1$ then the multiplicity of $R_{s}$ must not exceed two .

To show that the roots of the first characteristic equation satisfies the prior definition we assume that $t \in(0,1)$ and hence

$$
\rho(R)=\operatorname{det}\left[R A^{(0)}-A^{(t)}\right]=0
$$




$$
\begin{gathered}
\left.\rho(R)=\operatorname{det}\left[\begin{array}{cccc}
R & 0 & 0 & 0 \\
0 & R & 0 & 0 \\
0 & 0 & R & 0 \\
0 & 0 & 0 & R
\end{array}\right]-\left[\begin{array}{cccc}
0 & 1 & 0 & t h \\
0 & 1 & 0 & h \\
0 & 0 & 0 & 1 \\
0 & 0 & 0 & 1
\end{array}\right]\right]=0 \\
R^{2}(R-1)^{2}=0 \\
R_{1}=R_{2}=0 \\
R_{3}=R_{4}=1
\end{gathered}
$$

As a result, the developed method is zero stable.

\subsection{Convergence}

Theorem 3.1. (Henrici,1962) Consistency and zero stability are sufficient conditions for a linear multi step method to be convergent

The hybrid block method (8) is convergent since it satisfies both the consistency and zero stability conditions.

\section{Numerical Examples}

In this section accuracy of the general one-step implicit hybrid block method (8) with order 6 is tested on three experimental problems for the three cases simultaneously, with a fixed step size $h=\frac{5}{1000}$ for the first problem $h=\frac{1}{100}$ for the second and $h=\frac{0.1}{32}$ for the third. The computed results are then compared with recent methods and the new methods is found to have advantages as shown in Tables I-III.

Problem (1): $f\left(x, y, y^{\prime}\right)=3 y^{\prime}+8 e^{2 x}, \quad y(0)=1, \quad y^{\prime}(0)=1$.

Exact Solution : $y=-4 e^{2 x}+3 e^{3 x}+2$ with $h=\frac{5}{1000}$.

Source : [2].

Table I : Comparison of the proposed method with A.M. Badmus (2014).

\begin{tabular}{|l|l|l|l|l|}
\hline X VALUE & $\begin{array}{l}\text { ERROR FOR } \\
t=\frac{1}{3}\end{array}$ & $\begin{array}{l}\text { ERROR FOR } \\
t=\frac{1}{2}\end{array}$ & $\begin{array}{l}\text { ERROR FOR } \\
t=\frac{2}{3}\end{array}$ & $\begin{array}{l}\text { ERROR } \\
\text { AMB }\end{array}$ \\
\hline 0.0050000 & $4.440892 \mathrm{E}(-16)$ & $4.440892 \mathrm{E}(-16)$ & $2.220446 \mathrm{E}(-16)$ & $3.159 \mathrm{E}(-07)$ \\
\hline 0.0100000 & $8.881784 \mathrm{E}(-16)$ & $8.881784 \mathrm{E}(-16)$ & $6.661338 \mathrm{E}(-16)$ & $1.2709 \mathrm{E}(-06)$ \\
\hline 0.0150000 & $2.220446 \mathrm{E}(-16)$ & $2.220446 \mathrm{E}(-16)$ & $4.440892 \mathrm{E}(-16)$ & $8.6554 \mathrm{E}(-06)$ \\
\hline 0.0200000 & $6.661338 \mathrm{E}(-16)$ & $6.661338 \mathrm{E}(-16)$ & $8.881784 \mathrm{E}(-16)$ & $2.59148 \mathrm{E}(-05)$ \\
\hline 0.0250000 & $4.440892 \mathrm{E}(-16)$ & $4.440892 \mathrm{E}(-16)$ & $8.881784 \mathrm{E}(-16)$ & $3.395058 \mathrm{E}(-05)$ \\
\hline 0.0300000 & $1.332268 \mathrm{E}(-15)$ & $1.554312 \mathrm{E}(-15)$ & $2.664535 \mathrm{E}(-15)$ & $5.990417 \mathrm{E}(-05)$ \\
\hline 0.0400000 & $1.776357 \mathrm{E}(-15)$ & $1.998401 \mathrm{E}(-15)$ & $3.774758 \mathrm{E}(-15)$ & $8.885833 \mathrm{E}(-05)$ \\
\hline
\end{tabular}

Remark: AMB is the error in [2] . 
Problem (2): $f(x, y, z)=x\left(y^{\prime}\right)^{2}, y(0)=1, y^{\prime}(0)=\frac{1}{2}$.

Exact Solution : $y=1+\ln \left(\frac{2+x}{2-x}\right)$ with $h=\frac{1}{100}$.

Source : [1].

Table II : Comparison of the proposed method with Adetola Olaide (2013).

\begin{tabular}{|l|l|l|l|l|}
\hline X VALUE & $\begin{array}{l}\text { ERROR FOR } \\
t=\frac{1}{3}\end{array}$ & $\begin{array}{l}\text { ERROR FOR } \\
t=\frac{1}{2}\end{array}$ & $\begin{array}{l}\text { ERROR FOR } \\
t=\frac{2}{3}\end{array}$ & $\begin{array}{l}\text { ERROR } \\
\text { FOR EAO }\end{array}$ \\
\hline 0.1000000 & $2.220446 \mathrm{E}(-16)$ & $2.220446 \mathrm{E}(-16)$ & $6.661338 \mathrm{E}(-16)$ & $9.992 \mathrm{E}(-15)$ \\
\hline 0.2000000 & $0.000000 \mathrm{E}(+00)$ & $2.220446 \mathrm{E}(-16)$ & $1.332268 \mathrm{E}(-15)$ & $8.149 \mathrm{E}(-14)$ \\
\hline 0.3000000 & $8.881784 \mathrm{E}(-16)$ & $2.220446 \mathrm{E}(-16)$ & $4.440892 \mathrm{E}(-16)$ & $4.700 \mathrm{E}(-12)$ \\
\hline 0.4000000 & $1.998401 \mathrm{E}(-15)$ & $4.440892 \mathrm{E}(-16)$ & $1.332268 \mathrm{E}(-15)$ & $1.637 \mathrm{E}(-12)$ \\
\hline 0.5000000 & $3.330669 \mathrm{E}(-15)$ & $2.442491 \mathrm{E}(-15)$ & $3.774758 \mathrm{E}(-15)$ & $4.664 \mathrm{E}(-12)$ \\
\hline 0.6000000 & $8.659740 \mathrm{E}(-15)$ & $6.661338 \mathrm{E}(-15)$ & $1.065814 \mathrm{E}(-14)$ & $1.116 \mathrm{E}(-11)$ \\
\hline 0.7000000 & $1.798561 \mathrm{E}(-14)$ & $1.576517 \mathrm{E}(-14)$ & $2.642331 \mathrm{E}(-14)$ & $2.501 \mathrm{E}(-11)$ \\
\hline 0.8000000 & $3.819167 \mathrm{E}(-14)$ & $3.419487 \mathrm{E}(-14)$ & $5.861978 \mathrm{E}(-14)$ & $5.215 \mathrm{E}(-11)$ \\
\hline 0.9000000 & $7.971401 \mathrm{E}(-14)$ & $7.260859 \mathrm{E}(-14)$ & $1.265654 \mathrm{E}(-13)$ & $1.076 \mathrm{E}(-11)$ \\
\hline 1.0000000 & $1.665335 \mathrm{E}(-13)$ & $1.540990 \mathrm{E}(-13)$ & $2.711165 \mathrm{E}(-13)$ & $2.170 \mathrm{E}(-10)$ \\
\hline
\end{tabular}

Remark: EAO is the error in [1] .

$\operatorname{Problem}(3): y^{\prime \prime}+\frac{6}{x} y^{\prime}+\frac{6}{x^{2}} y=0, \quad y(1)=1, \quad y^{\prime}(1)=1$.

Exact Solution : $y=\frac{5}{3 x}-\frac{2}{3 x^{4}}$ with $h=\frac{0.1}{32}$.

Source : [3].

Table III : Comparison of the proposed method with A.M .Badmus (2014).

\begin{tabular}{|l|l|l|l|l|}
\hline X VALUE & $\begin{array}{l}\text { ERROR FOR } \\
t=\frac{1}{3}\end{array}$ & $\begin{array}{l}\text { ERROR FOR } \\
t=\frac{1}{2}\end{array}$ & $\begin{array}{l}\text { ERROR FOR } \\
t=\frac{2}{3}\end{array}$ & $\begin{array}{l}\text { ERROR } \\
\text { FOR EAM }\end{array}$ \\
\hline 1.0031250 & $2.220446 \mathrm{E}(-16)$ & $2.220446 \mathrm{E}(-16)$ & $2.220446 \mathrm{E}(-16)$ & $8.3 \mathrm{E}(-8)$ \\
\hline 1.0062500 & $0.000000 \mathrm{E}(+00)$ & $2.220446 \mathrm{E}(-16)$ & $4.440892 \mathrm{E}(-16)$ & $1.16 \mathrm{E}(-6)$ \\
\hline 1.0093750 & $2.220446 \mathrm{E}(-16)$ & $4.440892 \mathrm{E}(-16)$ & $8.881784 \mathrm{E}(-16)$ & $6.638 \mathrm{E}(-6)$ \\
\hline 1.0125000 & $8.881784 \mathrm{E}(-16)$ & $1.110223 \mathrm{E}(-15)$ & $1.776357 \mathrm{E}(-15)$ & $9.491 \mathrm{E}(-6)$ \\
\hline 1.0156250 & $1.332268 \mathrm{E}(-15)$ & $1.554312 \mathrm{E}(-15)$ & $3.108624 \mathrm{E}(-15)$ & $1.9535 \mathrm{E}(-6)$ \\
\hline 1.0187500 & $2.442491 \mathrm{E}(-15)$ & $2.664535 \mathrm{E}(-15)$ & $4.884981 \mathrm{E}(-15)$ & $9.416 \mathrm{E}(-6)$ \\
\hline 1.0218750 & $3.996803 \mathrm{E}(-15)$ & $3.996803 \mathrm{E}(-15)$ & $6.883383 \mathrm{E}(-15)$ & $4.6505 \mathrm{E}(-5)$ \\
\hline 1.0250000 & $5.107026 \mathrm{E}(-15)$ & $5.107026 \mathrm{E}(-15)$ & $8.659740 \mathrm{E}(-15)$ & $4.7122 \mathrm{E}(-5)$ \\
\hline 1.0281250 & $6.217249 \mathrm{E}(-15)$ & $5.995204 \mathrm{E}(-15)$ & $1.065814 \mathrm{E}(-14)$ & $1.86926 \mathrm{E}(-4)$ \\
\hline 1.0312500 & $7.327472 \mathrm{E}(-15)$ & $7.327472 \mathrm{E}(-15)$ & $1.287859 \mathrm{E}(-14)$ & $4.43321 \mathrm{E}(-4)$ \\
\hline
\end{tabular}

Remark: EAM is the error in [3]

\section{Conclusion}

A general one-step hybrid (GOHBM) block method with one off step point of order 6 has been successfully developed for the direct solution of general second order IVP. The developed method is tested on $t=\left\{\frac{1}{3}, \frac{1}{2}, \frac{2}{3}\right\}$. Numerical analysis shows that the developed method is consistent and zero stable which implies its convergence. Apart from having good properties of the numerical 
method, the numerical results suggest that the new method has not only out performed the existing methods, but also circumvent Dahlquists barrier.

\section{References}

[1] Adetola Olaide, Mattew Remilekun Odekunle and Mfon Odo Udoh, Four Steps Continuous Method for the Solution of $\mathrm{y}^{\prime \prime}=\mathrm{f}\left(\mathrm{x}, \mathrm{y}, \mathrm{y}{ }^{\prime}\right)$, American Journal of Computational Mathematics, 3 (2013), no. 2, 169-174.

http://dx.doi.org/10.4236/ajcm.2013.32025

[2] A.M. Badmus, An Efficient Seven-point Hybrid Block Method for the Direct Solution of $\mathrm{y}^{\prime \prime}=\mathrm{f}\left(\mathrm{x}, \mathrm{y}, \mathrm{y}^{\prime}\right)$, British Journal of Mathematics and Computer Science, 4 (2014), no. 19, 2840-2852.

http://dx.doi.org/10.9734/bjmcs/2014/6749

[3] A.M. Badmus, A New Eighth Order Implicit Block Algorithms for the Direct Solution of Second Order Ordinary Differential Equations, American Journal of Computational Mathematics, 4 (2014), no. 4, 376-386.

http://dx.doi.org/10.4236/ajcm.2014.44032

[4] C.W. Gear, Hybrid methods for initial value problems in ordinary differential equations, SIAM Journal of Numerical Analysis, 2 (1965), no. 1, 69-86. http://dx.doi.org/10.1137/0702006

[5] F. Ngwane, S. Jator, Block hybrid-second derivative method for stiff systems, Int. J. Pure Appl. Math, 80 (2012), no. 4, 543 - 559.

[6] G.G. Dahlquist, Numerical integration of ordinary differential equations, Math. Scand., 4 (1956), 69 - 86.

[7] J.D. Lambert, Computational Methods in Ordinary Differential Equations, John Wiley, New York, 1973.

[8] J.B. Rosser, A Runge-kutta for all seasons, SIAM, Rev., 9 (1967), no. 3, 417 - 452. http://dx.doi.org/10.1137/1009069

[9] L.F. Shampine, H. A. Watts, Block implicit one-step methods, Mathematics of Computation, 23 (1969), no. 108, 731-740.

http://dx.doi.org/10.1090/s0025-5718-1969-0264854-5

[10] P. Henrici, Discrete Variable Methods in ODEs, Wiley, New York, 1962.

[11] R.K. Sahi, S.N. Jator, N. A. Khan, A simpson's-type second derivative method for stiff systems, International Journal of Pure and Applied Mathematics, 81 (2012), no. 4, 619 - 633. 
[12] S.N. Jator, Solving second order initial value problems by a hybrid multistep method without predictors, Applied Mathematics and Computation, 217 (2010), no. 8, 4036 - 4046.

http://dx.doi.org/10.1016/j.amc.2010.10.010

[13] S.O. Fatunla, Numerical Methods for Initial Value Problems in Ordinary Differential Equation, Academic Press, New-York, 1988.

[14] W.E. Milne, Numerical Solution of Differential Equations, John Wiley and Sons, 1953.

[15] W. Gragg, H.J. Stetter, Generalized multistep predictor-corrector methods, J. Assoc. Comput. Mach., 11 (1964), no. 2, 188 - 209.

http://dx.doi.org/10.1145/321217.321223

[16] W.H. Enright, Second derivative multistep methods for stiff ordinary differential equations, SIAM J. Numer. Anal., 11 (1974), no. 2, 321 - 331. http://dx.doi.org/10.1137/0711029

Received: November 6, 2015; Published: February 8, 2016 